\title{
Long non-coding RNA XLOC_006390 promotes cervical cancer proliferation and metastasis through the regulation of SET domain containing 8
}

\author{
XIAOTIAN LUAN and YANKUI WANG \\ Department of Obstetrics and Gynecology, The Affiliated Hospital of Qingdao University, \\ Qingdao, Shandong 266003, P.R. China
}

Received August 25, 2016; Accepted October 24, 2016

DOI: $10.3892 /$ or.2017.5663

\begin{abstract}
The long non-coding RNA (lncRNA) XLOC_006390 is increased in various human cancer tissues and it plays important roles in cell growth and migration. However, the role of lncRNA XLOC_006390 in the progression and metastasis of cervical cancer has not been evaluated and remains unclear. In the present study, we hypothesized that IncRNA XLOC_006390 is also increased in cervical cancer, and upregulation of lncRNA XLOC_006390 contributes to cervical cancer metastasis. The expression of 1ncRNA XLOC_006390 in cervical cancer tissues and cell lines was analyzed using quantitative reversetranscription-polymerase chain reaction (qRT-PCR). RNA interference approach and an overexpression system were used to investigate the cellular functions of XLOC_006390 and SET domain containing 8 (SET8). Cell Counting Kit-8 (CCK-8) assay was performed to detect cell proliferation. Cell migration and invasion abilities were evaluated by Transwell assays. Western blotting and immunofluorescence were performed to detect SET8 protein expression. The results revealed that XLOC_006390 was increased in cervical cancer tissues. Patients with high XLOC_006390 expression were associated with FIGO stages III and IV $(\mathrm{P}=0.0170)$, lymphatic metastasis $(\mathrm{P}=0.0078)$ and distant metastasis $(\mathrm{P}=0.0025)$. Furthermore, SET8 was also increased in cervical cancer tissues and its expression was positively associated with XLOC_006390, and XLOC_006390 regulated SET8 expression. In addition, knockdown or overexpression of XLOC_006390 and SET8 expression suppressed or promoted cervical cancer cell proliferation, migration and invasion in vitro, respectively. In conclusion, our data suggest that lncRNA XLOC_006390 promotes cervical cancer cell growth and metastasis through the regulation of SET8, at
\end{abstract}

Correspondence to: Professor Yankui Wang, Department of Obstetrics and Gynecology, The Affiliated Hospital of Qingdao University, Qingdao, Shandong 266003, P.R. China E-mail:drwyk@outlook.com; yankuiwang2000@126.com

Key words: cervical cancer, long non-coding RNA, XLOC_006390, SET8, metastasis least partly, which indicate the critical roles of XLOC_006390 and SET8 in cervical cancer progression and metastasis.

\section{Introduction}

Cervical cancer is the second most common malignancy in women worldwide (1), and it leads to 266,000 cancer-related deaths among women worldwide (2). More than 52,000 new cervical cancer cases are diagnosed every year worldwide (2). Recently, with the development and effectiveness of early screening tests for cervical cancer, the incidence and mortality rates of cervical cancer in developed countries have decreased $(3,4)$. However, more than $80 \%$ of all new cervical cancer cases are diagnosed in developing countries (5), and cervical cancer metastasis negatively impacts the survival of affected cervical cancer patients directly $(3,4)$. Numerous studies, in recent years (6-8), have focused on the metastasis of cervical cancer, but the underlying molecular and cellular mechanisms remain confusing.

Long non-coding RNAs (lncRNAs) are a group of endogenous non-coding transcripts with more than 200 nucleotides in length $(9,10)$. For a long period of time, scientists believed that IncRNAs had no cellular and molecular functions, and were considered to be transcriptional noise. However, recently numerous studies indicate that lncRNAs contribute to different cellular processes (11-13). During the last decade, scientists have demonstrated that abnormal expression of lncRNAs is associated with human diseases (14-16), including different types of human cancers (17-19). The expression of lncRNA LINC01133 was decreased in human colorectal cancer (CRC) tissues and a low expression level of LIINC01133 was associated with poor survival in CRC patients. Overexpression of LINC01133 inhibited epithelial-mesenchymal transition (EMT) and metastasis by regulating SRSF6 (20). IncRNA colon cancer-associated transcript 2 (CCAT2) was found to be increased in human small cell lung cancer (SCLC) tissues and knockdown of CCAT2 expression suppressed the ability of SCLC cell proliferation and metastasis (21). lncRNA taurine upregulated gene 1 (TUG1) was found to be significantly increased in human liver cancer tissues and metastatic liver cancer cell lines and knockdown of TUG1 suppressed liver cancer growth, metastasis and angiogenesis through the 
regulation of miR-34a-5p and the VEGFA regulatory network in vitro and in vivo (22). Wang et al performed lncRNA microarray and analyzed the different expression levels of lncRNAs in pancreatic cancer and para-carcinoma tissues, and found that lncRNA XLOC_006390 was significantly increased in tumor tissues (23). IncRNA XLOC_006390 also called lncRNA HOXA13 was markedly increased in hepatocellular carcinoma (HCC), and its expression level was associated with HCC patient clinical progression and survival outcome (24). However, the role of IncRNA XLOC_006390 in the progression and metastasis of cervical cancer has not been evaluated and remains unclear. In the present study, we hypothesized that lncRNA XLOC_006390 is also increased in cervical cancer, and upregulation of IncRNA XLOC_006390 contributes to cervical cancer metastasis.

In the present study, we performed quantitative realtime polymerase chain reaction (qRT-PCR) and analyzed the expression level of lncRNA XLOC_006390 in human cervical cancer tissues. Our results found that lncRNA XLOC_006390 was significantly overexpressed in cervical cancer tissues compared with matched para-carcinoma tissues, which is consistent with findings of lncRNA microarray in pancreatic cancer (23). Furthermore, we confirmed that SET domain containing 8 (SET8) was increased in cervical cancer tissues and it was positively associated with the expression level of lncRNA XLOC_006390. In addition, knockdown of lncRNA XLOC_006390 and SET8 suppressed cervical cancer cell proliferation and metastasis in vitro. Our results suggest that XLOC_006390 promotes cervical cancer proliferation and migration through the regulation of SET8 expression, at least partly, which indicate critical roles of XLOC_006390 and SET8 in cervical cancer progression and metastasis.

\section{Materials and methods}

Cervical cancer tissues. Thirty-seven primary cervical cancer tissues and 37 matched adjacent para-carcinoma tissues were obtained from patients with cervical cancer through surgery at the Department of Obstetrics and Gynecology of The Affiliated Hospital of Qingdao University from 2015-2016. All of the patients who participated in the present study had never received preoperative radiotherapy and/or chemotherapy. The volunteers were pathologically diagnosed with infiltrating carcinoma. The tumor stage was confirmed by two gynecological oncologists based on histopathological evaluation according to the International Federation of Gynecology and Obstetrics (FIGO) staging system for cervical cancer. Clinicopathological data are available for all samples in Table I.

Cell lines and culture conditions. Five cervical cancer cell lines, SiHa, HeLa, Caski, C4-1 and C-33a, were purchased from the Cell Bank of the Chinese Academy of Sciences (Shanghai, China) and the American Type Culture Collection (ATCC; Manassas, VA, USA), respectively. All cell lines were cultured in RPMI-1640 medium supplemented with $10 \%$ fetal bovine serum (FBS) (both from Gibco-BRL, Gaithersburg, MD, USA). All the media contained $1 \%$ penicillin-streptomycin (100 U/ml penicillin and $100 \mu \mathrm{g} / \mathrm{ml}$ streptomycin).
All cervical cancer cells were cultured and maintained in a humidified incubator with $5 \% \mathrm{CO}_{2}$ at $37^{\circ} \mathrm{C}$.

Cell transfection. The $\mathrm{SiHa}$ cell line was used to perform siRNA knockdown experiments and 4C-1 cells were used in an overexpression system. Cells $\left(1 \times 10^{5}\right)$ were seeded into a 24-well culture plate, and incubated in RPMI-1640 medium with $10 \%$ FBS for $24 \mathrm{~h}$. Then, the cells were transfected with siRNA (100 nM) targeting XLOC_006390 (siRNA sense, UUCCUAAAAGCUCAGAAACAGUU and antisense, GUU UCUGAGCUUUUAGGAAGUUU); or SET8 (siRNA sense, AGGAAUAGAUCUUUGACUGCCUU and antisense, CAGU CAAAGAUCUAUUCCUACUU); or IncRNA XLOC_006390 overexpression vector $(20 \mu \mathrm{g})$ or SET8 overexpression plasmids $(20 \mu \mathrm{g})$ (both from Vipotion, Guangzhou, China) using Lipofectamine 2000 (Invitrogen, Carlsbad, CA, USA) in RPMI-1640 medium without FBS in accordance with the manufacturer's guidelines.

$q R T-P C R$. Total RNA was extracted from tissues and cells using TRIzol reagent (Invitrogen). The RNA concentration and quality were determined by NanoDrop 2000 (Quawell, San Jose, CA, USA). Total RNA ( $2 \mu \mathrm{g})$ was used for first strand cDNA synthesis with a reverse transcription reaction using a reverse transcription kit (Takara, Dalian, China). The corresponding cDNA was used for quantitative real-time PCR using SYBR-Green Real-Time Master Mix (Takara). GAPDH was used as the internal control. The primers used for XLOC_006390 were: 5'-CCTTTGAATCCCTGAGAACTG AAC-3' (forward) and 5'-ACCTTCCTTCCCACTGGACCT TC-3' (reverse); for SET8, 5'-ACTTACGGATTTCTACCC TGT-3' (forward) and 5'-CGATGAGGTCAATCTTC-3' (reverse); for GAPDH, 5'-CCCACTCCTCCACCTTTGAC-3' (forward) and 5'-ATACCAGGAAATGAGCTTGACAA-3' (reverse). The qRT-PCR analysis was performed on Applied Biosystems 7500 Sequence Detection System (ABI, Foster City, CA, USA). Data were analyzed using the $2^{-\Delta \Delta \mathrm{Ct}}$ method.

Western blotting. Total protein from tissues and cells were extracted using SDS lysis buffer (Beyotime, Shanghai, China). Tissues and cells were homogenized and incubated on ice for $10 \mathrm{~min}$ and centrifuged at $12,000 \mathrm{x} \mathrm{g}$ for $15 \mathrm{~min}$. The supernatant was then collected and its concentration was determined using a BCA protein assay kit (Pierce, Rockford, IL, USA). Total protein $(20 \mu \mathrm{g})$ was separated on $12 \%$ SDS polyacrylamide gels and transferred to polyvinylidene difluoride (PVDF) membranes (Millipore, Billerica, MA, USA) in $25 \mathrm{mM}$ Tris-base and $190 \mathrm{mM}$ glycine at $60 \mathrm{~V}$ for $3 \mathrm{~h}$ at $4^{\circ} \mathrm{C}$. The membranes were blocked at $4^{\circ} \mathrm{C}$ in Tris-buffered saline (TBS) containing 5\% non-fat dried milk overnight and washed with phosphate-buffered saline (PBS) three times. Then, the membranes were incubated with primary antibodies (SET8; 1:10,000; ab177488) (GAPDH; 1:2,500; ab9485) (both from Abcam, Cambridge, MA, USA) for $1 \mathrm{~h}$ at room temperature and washed with PBS in triplicate. Finally, the membranes were cultured with goat anti-rabbit IgG-HRP (sc2004; Santa Cruz Biotechnology, Santa Cruz, CA, USA) at a 1:5,000 dilution for $1 \mathrm{~h}$ at $37^{\circ} \mathrm{C}$. Proteins were analyzed by enhanced chemiluminescence (ECL) as described by the manufacturer's instructions (Beyotime). 
Table I. The association between XLOC_006390 expression and clinicopathological factors in cervical cancer patients.

\begin{tabular}{lcccc}
\hline & \multicolumn{3}{c}{ XLOC_006390 } & \\
\cline { 3 - 4 } Clinical parameter & Total & High (\%) & Low (\%) & P-value \\
\hline Age (years) & & & & 0.5148 \\
$\leq 40$ & 17 & 7 & 10 & \\
$>40$ & 20 & 11 & 9 & \\
Size (cm) & & & & 0.3155 \\
$\geq 4$ & 13 & 8 & 5 & \\
$<4$ & 24 & 10 & 14 & \\
FIGO stages & & & & 0.0170 \\
I-II & 14 & 3 & 11 & \\
III-IV & 23 & 15 & 8 & \\
Lymphatic metastasis & & & & 0.0078 \\
Yes & 27 & 17 & 10 & \\
$\quad$ No & 10 & 1 & 9 & \\
Distant metastasis & & & & 0.0025 \\
Yes & 21 & 15 & 6 & \\
No & 16 & 3 & 13 & \\
\hline
\end{tabular}

FIGO, International Federation of Gynecology and Obstetrics. ${ }^{\mathrm{a}} \mathrm{Chi}-$ squared test.

Immunofluorescence (IF). For IF, SiHa or C4-1 cells were transfected with SET8-siRNA or SET8 overexpression vectors and cultured on a coverslip for $48 \mathrm{~h}$. Then, the coverslips were washed with PBS for 5 min and fixed in 4\% paraformaldehyde (Beyotime). Subsequently, the cells were washed with PBS in triplicate and incubated with a primary antibody against SET8 at $4^{\circ} \mathrm{C}$ for $12 \mathrm{~h}$. The cells were then incubated with Alexa Fluor 594-conjugated goat anti-rabbit secondary antibody (Thermo Fisher Scientific, Inc., Waltham, MA, USA). 4',6-Diamidino-2-phenylindole (DAPI) was used to stain the nucleus. Images were captured by an FV10i confocal microscope (Olympus, Tokyo, Japan).

Cell Counting Kit-8 (CCK-8) assay of cell proliferation. Following transfection with siRNA or overexpression vectors, $0.5 \times 10^{3} \mathrm{SiHa}$ or $\mathrm{C} 4-1$ cells were seeded in a 96 -well cell culture plate. At 24, 48 and $72 \mathrm{~h}$, cells were treated with $10 \mu \mathrm{l} \mathrm{CCK}-8$ at $37^{\circ} \mathrm{C}$ for $4 \mathrm{~h}$. The absorbance at $450 \mathrm{~nm}$ was assessed using a microplate reader Thermo Plate (Rayto Life and Analytical Sciences Co., Ltd., Shenzhen, China). All experiments were performed at least three times.

Migration and invasion assays. Transwell migration and invasion assays were performed as previously described (25). $\mathrm{SiHa}$ or C4-1 cells/well $\left(1.5 \times 10^{5}\right)$ were used for a migration assay, and $1 \times 10^{5} \mathrm{SiHa}$ or $\mathrm{C} 4-1$ cells/well were used for an invasion assay. Cells that migrated or invaded were fixed with $100 \%$ methanol for $30 \mathrm{~min}$ and stained using $0.5 \%$ crystal violet (Sigma-Aldrich, St. Louis, MO, USA) for $20 \mathrm{~min}$ and counted in five random fields of each filter under a microscope (IX71; Olympus) at a magnification of $\mathrm{x} 200$.
Statistical analysis. All data are presented as the mean \pm SD. SPSS 20.0 software (SPSS, Inc., Chicago, IL, USA) was used for statistical analysis. All experiments were performed at least three times. The statistical significance of differences between groups was assessed using one-way analysis of variance (ANOVA) or a paired/unpaired Student's t-test or a Chi-square test, and $\mathrm{P}<0.05$ was considered to indicate a statistically significant result.

\section{Results}

Expression level of IncRNA XLOC_006390 in cervical cancer tissues and cell lines. To investigate the expression level of lncRNA XLOC_006390 in human cervical cancer tissues, qRT-PCR was performed to detect the expression of XLOC_006390 in 37 cervical cancer samples. IncRNA XLOC_006390 was significantly upregulated in tumor tissues compared with matched adjacent para-carcinoma tissues (Fig. 1A). Furthermore, we also analyzed the expression of XLOC_006390 in five cervical cancer cell lines and the results revealed that XLOC_006390 was increased in SiHa cells, and decreased in C4-1 cells compared with the HeLa cell line (Fig. 1B). Based on the results, the SiHa and C4-1 cell lines were selected for further study.

Correlation of IncRNA XLOC_006390 expression with clinicopathological factors in cervical cancer patients. To investigate the relationship between IncRNA XLOC_006390 expression and clinicopathological factors in cervical cancer, 37 patients were divided into two groups according to the median expression level of XLOC_006390 (2.98): a low XLOC_006390 expression group ( $\mathrm{n}=19$, XLOC_006390 expression ratio $\leq$ median ratio) and a high XLOC_006390 expression group $(n=18$, XLOC_006390 expression ratio $\geq$ median ratio) (Fig. $1 \mathrm{C}$ ). The relationship between the expression level of XLOC_006390 and clinicopathological factors are shown in Table I. High XLOC_006390 expression was observed to be associated with FIGO stage $(\mathrm{P}=0.0170)$, lymphatic $(\mathrm{P}=0.0078)$ and distant metastasis $(\mathrm{P}=0.0025)$. In contrast, there was no association between XLOC_006390 expression with age $(\mathrm{P}=0.5148)$ and tumor size $(\mathrm{P}=0.3155)$.

Effects of XLOC_006390 on cervical cancer cell proliferation. To evaluate the role of IncRNA XLOC_006390 in cervical cancer cell proliferation, the expression of XLOC_006390 was knocked down or overexpressed by siRNA or lncRNA LXOC_006390 overexpression vector transfection, respectively. The expression of XLOC_006390 was decreased in the $\mathrm{SiHa}$ cells after transfection with XLOC_006390-siRNA (Fig. 2A) and increased in C4-1 cells after transfection with XLOC_006390 overexpression vectors (Fig. 2B). CCK-8 assay results revealed that suppression or overexpression of XLOC_006390 expression inhibited or promoted the proliferation of the SiHa and C4-1 cell lines, respectively (Fig. 2C and D).

Effects of XLOC_006390 on cervical cancer cell migration and invasion. To investigate the effect of lncRNA XLOC_006390 on cervical cancer cell migration and invasion, Transwell assays were performed. As shown in Fig. 3A and B, 
A

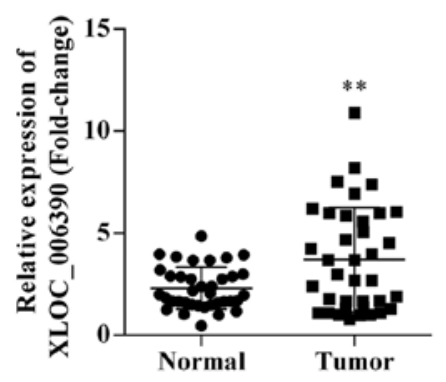

B

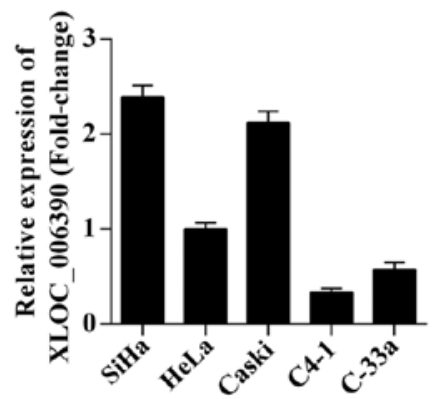

C

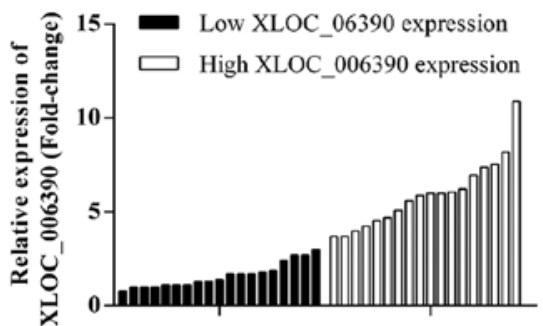

Figure 1. Expression level of lncRNA XLOC_006390 in cervical cancer tissues and cell lines. (A) qRT-PCR analysis determined the expression of lncRNA XLOC_006390 in cervical cancer tissues $(\mathrm{n}=37)$ ) ${ }^{* *} \mathrm{P}<0.01$. (B) qRT-PCR analysis determined the expression of IncRNA XLOC_006390 in five cervical cancer cell lines. (C) According to the median ratio of XLOC_006390 expression level (2.98) in tumor tissues, XLOC_006390 expression was classified into two groups: a low XLOC_006390 expression group ( $\mathrm{n}=19)$ and a high XLOC_006390 expression group ( $\mathrm{n}=18)$.

A

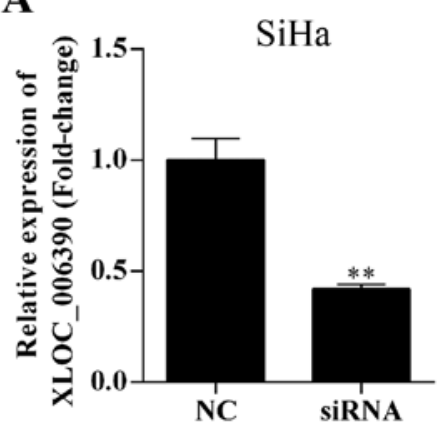

C

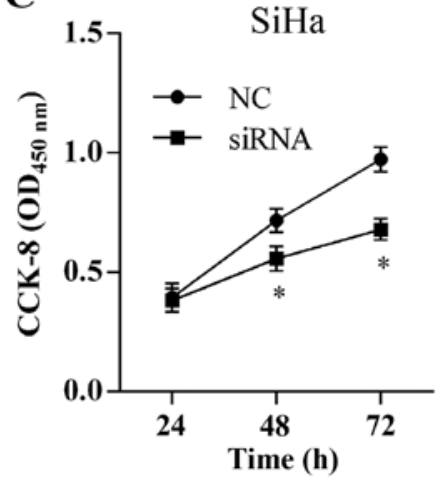

B

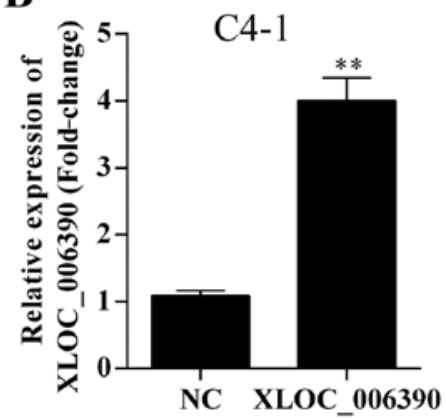

D

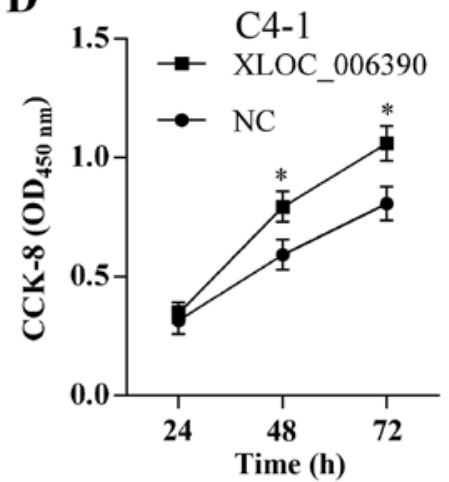

Figure 2. Effects of XLOC_006390 on cervical cancer cell proliferation. (A) qRT-PCR analysis determined the expression of XLOC_006390 in SiHa cells after transfection with XLOC_006390-siRNA; ${ }^{* *}$ P<0.01. (B) qRT-PCR analysis determined the expression of XLOC_006390 in C4-1 cells after transfection with XLOC_006390 overexpression vectors; ${ }^{* *} \mathrm{P}<0.01$. (C) CCK-8 assay results indicated that knockdown of XLOC_006390 suppressed SiHa cell proliferation; ${ }^{*} \mathrm{P}<0.05$. (D) CCK-8 assay results indicated that overexpression of XLOC_006390 promoted C4-1 cell proliferation; ${ }^{*} \mathrm{P}<0.05$. siRNA, XLOC_006390-siRNA; XLOC_006390, IncRNA XLOC_ 006390 overexpression vectors.

knockdown of XLOC_006390 inhibited the migration and invasion abilities of the SiHa cells. Overexpression of XLOC_006390 promoted the migration and invasion abilities of the $\mathrm{C} 4-1$ cells (Fig. 3C and D).

SET8 is upregulated in cervical cancer tissues and correlated with XLOC_006390 expression. The protein and mRNA expression of SET8 in cervical cancer tissues were detected using western blotting and qRT-PCR methods, respectively. It was observed that both the protein and mRNA expression level of SET8 were significantly increased in cervical cancer tissues compared with matched adjacent para-carcinoma tissues (Fig. 4A and B). Due to the fact that both the expression of XLOC_006390 and SET8 were significantly increased, we theorized a correlation between XLOC_006390 expression and SET8 expression in cervical cancer. The expression levels of SET8 in cervical cancer tissues were positively associated with the expression levels of XLOC_006390 ( $\mathrm{P}=0.0049$; $\mathrm{R}=0.2053$; Fig. 4C).

Effects of SET8 on cervical cancer cell proliferation. To evaluate the effects of SET8 on cervical cancer cell proliferation, the expression of the SET8 protein in $\mathrm{SiHa}$ and C4-1 cells after transfection with SET8-siRNA or SET8 
A

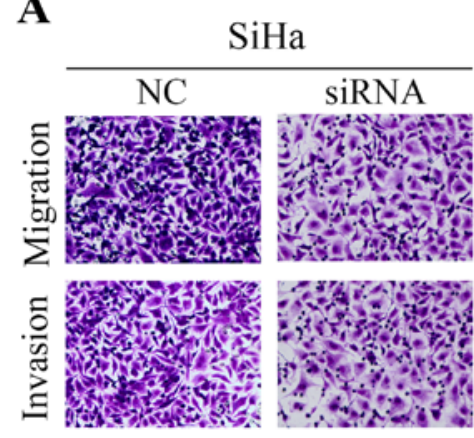

C

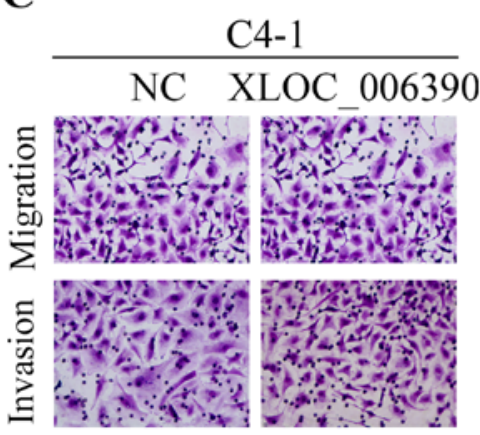

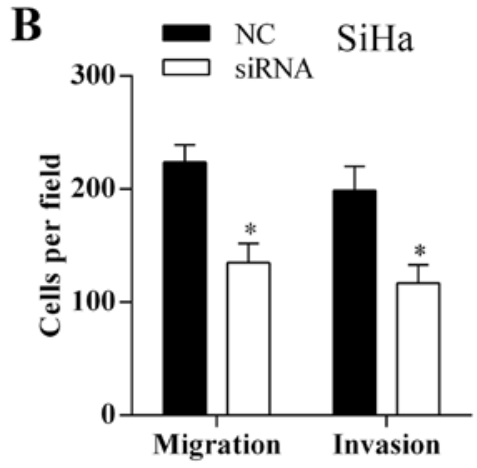

D

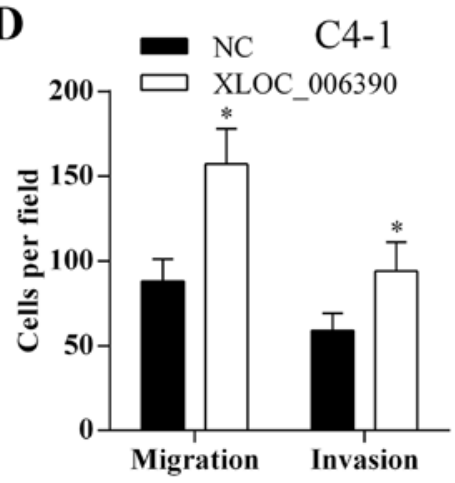

Figure 3. Effects of XLOC_006390 on cervical cancer cell migration and invasion. (A and C) Transwell assay was performed to detect the migration and invasion abilities of SiHa and C4-1 cells (magnification, x40). (B and D) The average migratory and invasive SiHa and C4-1 number of cells/field among different experimental groups. Data are presented as the mean \pm SD from three independent experiments; "P<0.05. siRNA, XLOC_006390-siRNA; XLOC_006390, IncRNA XLOC_006390 overexpression vectors.

A

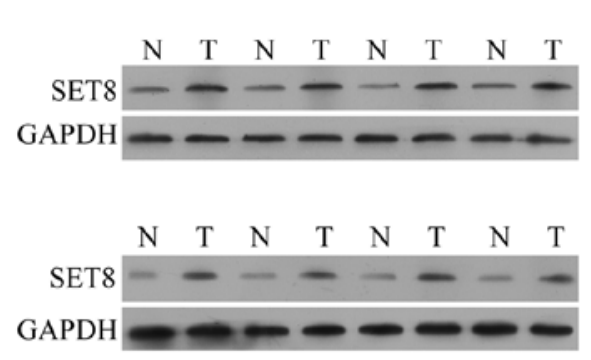

B

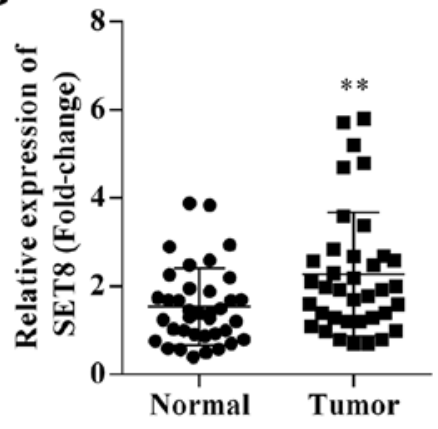

C

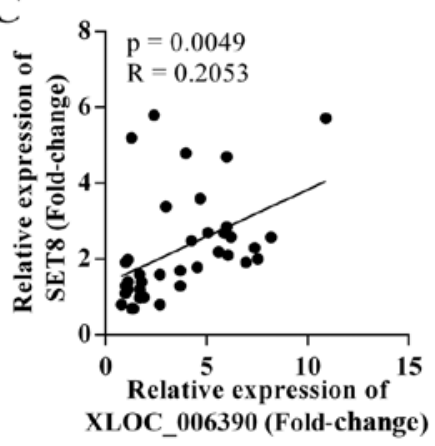

Figure 4. SET8 is upregulated in cervical cancer tissues and correlated with XLOC_006390 expression. (A) Western blotting analysis revealed the expression of SET8 in cervical cancer tissues. N, normal tissues, T, tumor tissues. (B) qRT-PCR analysis determined the expression of SET8 in cervical cancer tissues $(\mathrm{n}=37) ;{ }^{* *} \mathrm{P}<0.01$. (C) The expression levels of SET8 were positively correlated with the expression levels of XLOC_006390 (P=0.0049, $\left.\mathrm{R}=0.2053\right)$.

overexpression vectors were examined by western blotting and IF. Firstly, as shown in Fig. 4C, we found that the expression level of SET8 was positively associated with XLOC_006390 expression. In the present experiment we found that SET8 was decreased or increased after transfection with XLOC_006390-siRNA or XLOC_006390 overexpression vectors in SiHa or C4-1 cells, respectively (Fig. 5A and B). Furthermore, western blotting and IF results revealed that the expression of SET8 was suppressed or increased in SiHa or C4-1 cells after transfection with SET8-siRNA or SET8 overexpression vectors, respectively (Fig. 5A-C). In addition, CCK-8 assay results revealed that suppressed SET8 expression inhibited the proliferation of $\mathrm{SiHa}$ cells and overexpression of XLOC_006390 promoted C4-1 cell proliferation (Fig. 5D).
Effects of SET8 on cervical cancer cell migration and invasion. To investigate the role of SET8 on cervical cancer cell migration and invasion, Transwell assays were performed. As shown in Fig. 6, knockdown or overexpression of SET8 inhibited or promoted the migration and invasion abilities of the $\mathrm{SiHa}$ and $\mathrm{C} 4-1$ cells, respectively.

\section{Discussion}

Cervical cancer is still one of the leading causes of cancerrelated deaths in females worldwide (26). Although, the treatment methods including surgery, radiotherapy and chemotherapy have been greatly improved, the survival rate of cervical cancer patients is still unsatisfactory due to the high 
A

\begin{tabular}{rrrrrr}
\cline { 3 - 4 } \multicolumn{1}{c}{ NC } & SiRNA & & \\
SET8 & & & SET8 & NC & S-siRNA \\
GAPDH & & & GAPDH & & \\
\hline
\end{tabular}

B C4-1
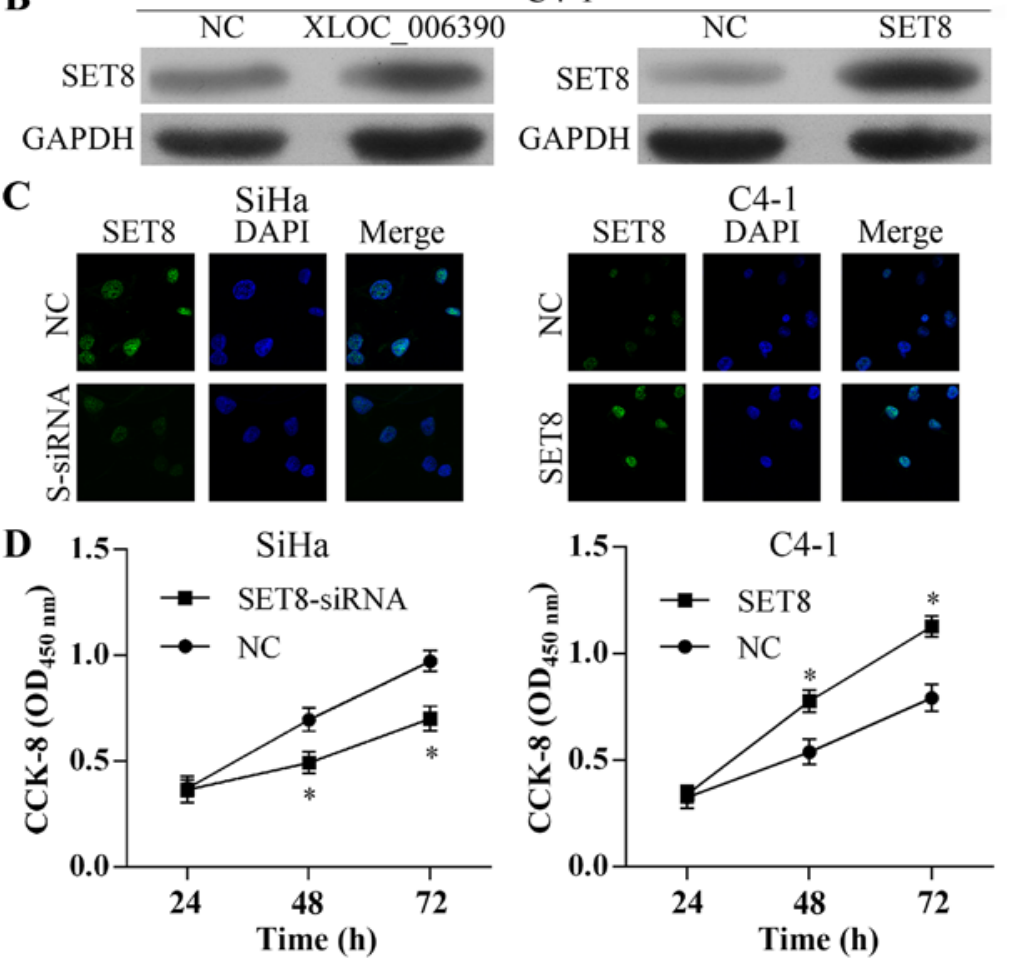

Figure 5. Effects of SET8 on cervical cancer cell proliferation. (A) Western blotting revealed the expression of SET8 in SiHa cells after transfection with XLOC_006390-siRNA and SET8-siRNA. siRNA, XLOC_006390-siRNA; S-siRNA, SET8-siRNA. (B) Western blotting revealed the expression of SET8 in C4-1 cells after transfection with XLOC_006390 overexpression and SET8 overexpression vectors. XLOC_006390, IncRNA XLOC_006390 overexpression vectors; SET8, SET8 overexpression vectors. (C) Immunofluorescence was used to analyze the expression of SET8 in SiHa and C4-1 cells after transfection with SET8-siRNA and SET8 overexpression vectors. (D) CCK-8 assay results indicated that knockdown of SET8 suppressed SiHa cell proliferation and overexpression of SET8 promoted C4-1 cell proliferation; ${ }^{*} \mathrm{P}<0.05$.
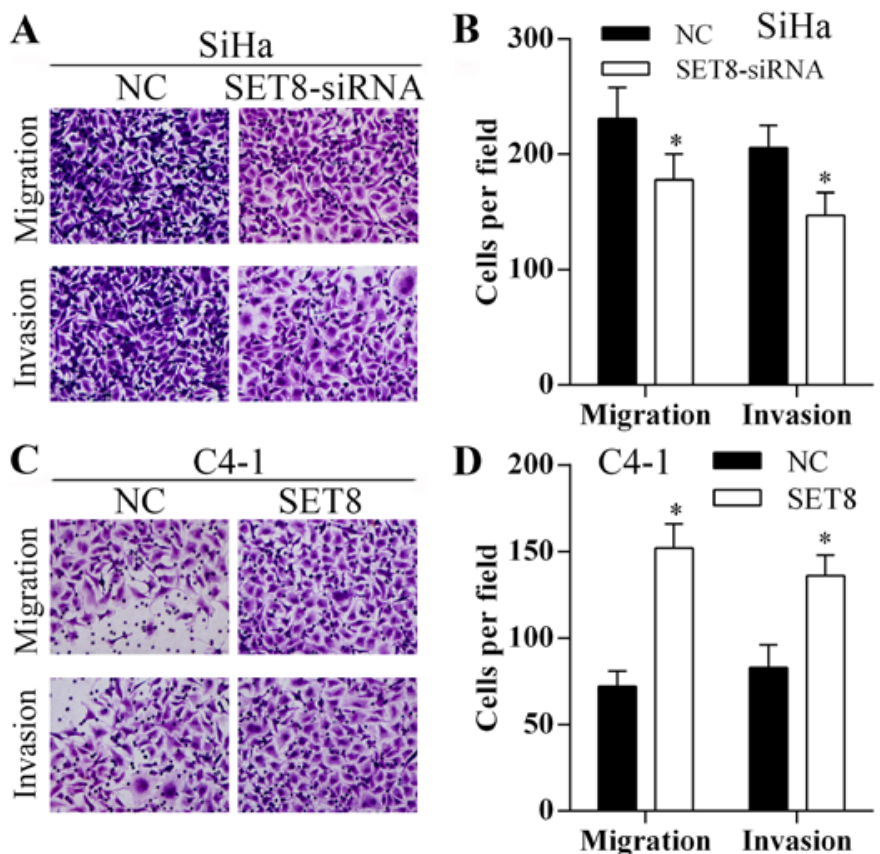

Figure 6. Effects of SET8 on cervical cancer cell migration and invasion. (A and C) Transwell assays were performed to detect the migration and invasion abilities of SiHa and C4-1 cells (magnification, x40). (B and D) The average migratory and invasive SiHa and C4-1 number of cells/field among different experimental groups. Data are presented as the mean \pm SD from three independent experiments; ${ }^{*} \mathrm{P}<0.05$. 
recurrence and metastasis rate $(3,4)$. Therefore, searching for a useful molecular biomarker may provide a new strategy to improve the survival of cervical cancer patients.

Numerous studies have indicated that lncRNAs play critical roles in various physiological processes, including cell proliferation, cell differentiation and epigenetic information (27-29). Increased research has revealed that dysregulated expression of IncRNAs is involved in pathological processes, such as malignant tumors $(30,31)$. Effective control of cell growth, survival and aggressiveness plays a critical role in preventing and treating tumors, successfully. Therefore, increased understanding of the biological functions of lncRNAs may provide potential useful approaches for cervical cancer diagnosis and treatment. The expression of 1ncRNA GAS5 was decreased in cervical cancer tissues and a lower expression of lncRNA GAS5 was associated with a poorer survival rate of patients (32). Cervical cancer cell proliferation was promoted by lncNRA CCHE1 through regulation of PCNA expression (33). lncRNA HOTAIR was increased in cervical cancer tissues and a higher expression of HOTAIR was associated with poorer prognosis. HOTAIR could promote cervical cancer cell aggressiveness through the promotion of VEGF and MMP-9 expression in vitro (34). However, there are no studies concerning lncRNA XLOC_006390 in the progression and metastasis of cervical cancer. In the present study, we found that lncRNA XLOC_006390 was increased in cervical cancer tissues and a higher expression of XLOC_006390 was associated with advanced FIGO stage, lymphatic metastasis and distant metastasis. Furthermore, knockdown of XLOC_006390 suppressed $\mathrm{SiHa}$ proliferation, migration and invasion in vitro, and overexpression of XLOC_006390 promoted C4-1 cell proliferation, migration and invasion. Moreover, SET domain containing 8 (SET8) was found to be overexpressed in cervical cancer tissues and its expression level was positively associated with XLOC_006390 expression. In addition, knockdown of XLOC_006390 suppressed SET8 expression, and overexpression of XLOC_006390 induced SET8 expression. Our results revealed that XLOC_006390 may play a critical role in the proliferation and metastatic potential of cervical cancer through the regulation of SET8 expression. Nevertheless, only 37 patients were involved in the present study, this may be a limitation for analyzing the correlation of lncRNA XLOC_006390 expression with clinicopathological factors in cervical cancer patients. More volunteers are needed for the investigation of the role of IncRNA XLOC_006390 in the progression and metastasis of cervical cancer, and to confirm the potential role of IncRNA XLOC_006390 as a diagnostic and treatment target for cervical cancer.

SET8 also called PR-Set7/9, KMT5A or SETD8, is one of the SET domain-containing methyltransferases that specifically targets histone H4 lysine 20 (H4K20) for monomethylation, and which exerts diverse biological processes, including activation or silencing of gene transcription $(35,36)$, maintaining chromosome structure and stability $(37,38)$, regulating the cell cycle and preventing premature chromatin compaction in the $\mathrm{S}$ phase $(39,40)$, and rescuing and degrading DNA damage $(41,42)$. Yu et al found that miR-7 suppressed the invasive potential of breast cancer cells by targeting SET8 (43). However, the role of SET8 in cervical cancer metastasis and how the expression of SET8 is regulated are poorly understood. In the present study, we found that SET8 was increased in cervical cancer tissues. Knockdown of SET8 suppressed SiHa proliferation, migration and invasion in vitro, and overexpression of SET8 promoted C4-1 cell growth and metastasis. Furthermore, the expression level of SET8 was positively associated with the expression of XLOC_006390. All the results indicated that upregulation of IncRNA XLOC_006390 may promote cervical cancer cell proliferation and metastasis through the regulation of SET8. Nevertheless, our results only revealed that knockdown of XLOC_006390 suppressed the expression SET8 and overexpression of XLOC_006390 induced the expression of SET8. How XLOC_006390 regulates SET8 expression and its regulatory mechanism need to be confirmed in the future.

In conclusion, we demonstrated that lncRNA XLOC_006390 and SET8 were increased in cervical cancer tissues, and the expression level of SET8 was positively associated with the expression of XLOC_006390. Furthermore, lncRNA XLCO_006390 regulated SET8 expression. Moreover, knockdown or overexpression of XLOC_006390 and SET8 expression suppressed or promoted cervical cancer cell proliferation and metastasis in vitro, respectively. In conclusion, our data demonstrated that IncRNA XLOC_006390 promoted cervical cancer cell growth and metastasis through the regulation of SET8, at least partly, which indicated the critical roles of XLOC_006390 and SET8 in cervical cancer progression and metastasis.

\section{References}

1. Torre LA, Bray F, Siegel RL, Ferlay J, Lortet-Tieulent J and Jemal A: Global cancer statistics, 2012. CA Cancer J Clin 65: 87-108, 2015

2. Ferlay J, Soerjomataram I, Dikshit R, Eser S, Mathers C, Rebelo M, Parkin DM, Forman D and Bray F: Cancer incidence and mortality worldwide: Sources, methods and major patterns in GLOBOCAN 2012. Int J Cancer 136: E359-E386, 2015.

3. Wang W, Jia HL, Huang JM, Liang YC, Tan H, Geng HZ, Guo LY and Yao SZ: Identification of biomarkers for lymph node metastasis in early-stage cervical cancer by tissue-based proteomics. Br J Cancer 110: 1748-1758, 2014.

4. Fleming ND, Frumovitz M, Schmeler KM, dos Reis R, Munsell MF, Eifel PJ, Soliman PT, Nick AM, Westin SN and Ramirez PT: Significance of lymph node ratio in defining risk category in node-positive early stage cervical cancer. Gynecol Oncol 136: 48-53, 2015.

5. Siegel R, Naishadham D and Jemal A: Cancer statistics, 2013. CA Cancer J Clin 63: 11-30, 2013.

6. Ying TH, Lee CH, Chiou HL, Yang SF, Lin CL, Hung CH, Tsai JP and Hsieh YH: Knockdown of Pentraxin 3 suppresses tumorigenicity and metastasis of human cervical cancer cells. Sci Rep 6: 29385, 2016.

7. Xin M, Qiao Z, Li J, Liu J, Song S, Zhao X, Miao P, Tang T, Wang L, Liu W, et al: miR-22 inhibits tumor growth and metastasis by targeting ATP citrate lyase: Evidence in osteosarcoma, prostate cancer, cervical cancer and lung cancer. Oncotarget 7: 44252-44265, 2016.

8. Zhou Q, Han LR, Zhou YX and Li Y: MiR-195 suppresses cervical cancer migration and invasion through targeting Smad3. Int J Gynecol Cancer 26: 817-824, 2016.

9. Mattick JS: The genetic signatures of noncoding RNAs. PLoS Genet 5: e1000459, 2009.

10. Rinn JL and Chang HY: Genome regulation by long noncoding RNAs. Annu Rev Biochem 81: 145-166, 2012.

11. Wang Y, Zhong H, Xie X, Chen CY, Huang D, Shen L, Zhang H, Chen ZW and Zeng G: Long noncoding RNA derived from CD244 signaling epigenetically controls $\mathrm{CD}^{+} \mathrm{T}$-cell immune responses in tuberculosis infection. Proc Natl Acad Sci USA 112: E3883-E3892, 2015 
12. Carlson HL, Quinn JJ, Yang YW, Thornburg CK, Chang HY and Stadler HS: LncRNA-HIT functions as an epigenetic regulator of chondrogenesis through its recruitment of p100/CBP complexes. PLoS Genet 11: e1005680, 2015.

13. Zhou C, York SR, Chen JY, Pondick JV, Motola DL, Chung RT and Mullen AC: Long noncoding RNAs expressed in human hepatic stellate cells form networks with extracellular matrix proteins. Genome Med 8: 31, 2016.

14. Schmitz SU, Grote P and Herrmann BG: Mechanisms of long noncoding RNA function in development and disease. Cell Mol Life Sci 73: 2491-2509, 2016

15. Takahashi K, Yan I, Haga $\mathrm{H}$ and Patel T: Long noncoding RNA in liver diseases. Hepatology 60: 744-753, 2014.

16. Chen Z, Luo Y, Yang W, Ding L, Wang J, Tu J, Geng B, Cui Q and Yang J: Comparison analysis of dysregulated LncRNA profile in mouse plasma and liver after hepatic ischemia/reperfusion injury. PLoS One 10: e0133462, 2015.

17. Li P, Zhang G, Li J, Yang R, Chen S, Wu S, Zhang F, Bai Y, Zhao $\mathrm{H}$, Wang Y, et al: Long noncoding RNA RGMB-AS1 indicates a poor prognosis and modulates cell proliferation, migration and invasion in lung adenocarcinoma. PLoS One 11: e0150790, 2016.

18. Li G, Zhang H, Wan X, Yang X, Zhu C, Wang A, He L, Miao R, Chen $\mathrm{S}$ and Zhao H: Long noncoding RNA plays a key role in metastasis and prognosis of hepatocellular carcinoma. Biomed Res Int 2014: 780521, 2014

19. Liu T, Zhang X, Yang YM, Du LT and Wang CX: Increased expression of the long noncoding RNA CRNDE-h indicates a poor prognosis in colorectal cancer, and is positively correlated with IRX5 mRNA expression. Onco Targets Ther 9: 1437-1448, 2016.

20. Kong J, Sun W, Li C, Wan L, Wang S, Wu Y, Xu E, Zhang H and Lai M: Long non-coding RNA LINC01133 inhibits epithelialmesenchymal transition and metastasis in colorectal cancer by interacting with SRSF6. Cancer Lett 380: 476-484, 2016.

21. Chen S, Wu H, Lv N, Wang H, Wang Y, Tang Q, Shao H and Sun C: LncRNA CCAT2 predicts poor prognosis and regulates growth and metastasis in small cell lung cancer. Biomed Pharmacother 82: 583-588, 2016.

22. Dong R, Liu GB, Liu BH, Chen G, Li K, Zheng S and Dong KR: Targeting long non-coding RNA-TUG1 inhibits tumor growth and angiogenesis in hepatoblastoma. Cell Death Dis 7: e2278, 2016.

23. Wang Y, Li Z, Zheng S, Zhou Y, Zhao L, Ye H, Zhao X, Gao W, Fu Z, Zhou Q, et al: Expression profile of long non-coding RNAs in pancreatic cancer and their clinical significance as biomarkers. Oncotarget 6: 35684-35698, 2015.

24. Quagliata L, Matter MS, Piscuoglio S, Arabi L, Ruiz C, Procino A, Kovac M, Moretti F, Makowska Z, Boldanova T, et al: Long noncoding RNA HOTTIP/HOXA13 expression is associated with disease progression and predicts outcome in hepatocellular carcinoma patients. Hepatology 59: 911-923, 2014.

25. Li J,Huang H, Li Y, Li L, Hou W and You Z: Decreased expression of long non-coding RNA GAS5 promotes cell proliferation, migration and invasion, and indicates a poor prognosis in ovarian cancer. Oncol Rep 36: 3241-3250, 2016.

26. Arbyn M, Castellsagué X, de Sanjosé S, Bruni L, Saraiya M, Bray $\mathrm{F}$ and Ferlay J: Worldwide burden of cervical cancer in 2008. Ann Oncol 22: 2675-2686, 2011.

27. Kotake Y, Nakagawa T, Kitagawa K, Suzuki S, Liu N, Kitagawa M and Xiong Y: Long non-coding RNA ANRIL is required for the PRC2 recruitment to and silencing of $p 15^{I N K 4 B}$ tumor suppressor gene. Oncogene 30: 1956-1962, 2011.
28. Liu Y, Zhao J, Zhang W, Gan J, Hu C, Huang G and Zhang Y: lncRNA GAS5 enhances G1 cell cycle arrest via binding to YBX1 to regulate p21 expression in stomach cancer. Sci Rep 5: 10159, 2015.

29. Pan Y, Li G, Zhong H, Chen M, Chen T, Gao L, Wu H and Guo J: RIG-I inhibits pancreatic $\beta$ cell proliferation through competitive binding of activated Src. Sci Rep 6: 28914, 2016.

30. Hrdlickova B, de Almeida RC, Borek Z and Withoff S: Genetic variation in the non-coding genome: Involvement of microRNAs and long non-coding RNAs in disease. Biochim Biophys Acta 1842: 1910-1922, 2014.

31. Kung JT, Colognori D and Lee JT: Long noncoding RNAs: Past, present, and future. Genetics 193: 651-669, 2013.

32. Cao S, Liu W, Li F, Zhao W and Qin C: Decreased expression of IncRNA GAS5 predicts a poor prognosis in cervical cancer. Int J Clin Exp Pathol 7: 6776-6783, 2014.

33. Yang M, Zhai X, Xia B, Wang Y and Lou G: Long noncoding RNA CCHE1 promotes cervical cancer cell proliferation via upregulating PCNA. Tumour Biol 36: 7615-7622, 2015.

34. Kim HJ, Lee DW, Yim GW, Nam EJ, Kim S, Kim SW and Kim YT: Long non-coding RNA HOTAIR is associated with human cervical cancer progression. Int J Oncol 46: 521-530, 2015.

35. Congdon LM, Houston SI, Veerappan CS, Spektor TM and Rice JC: PR-Set7-mediated monomethylation of histone H4 lysine 20 at specific genomic regions induces transcriptional repression. J Cell Biochem 110: 609-619, 2010.

36. Li Z, Nie F, Wang S and Li L: Histone H4 Lys 20 monomethylation by histone methylase SET 8 mediates Wnt target gene activation. Proc Natl Acad Sci USA 108: 3116-3123, 2011.

37. Houston SI, McManus KJ, Adams MM, Sims JK, Carpenter PB, Hendzel MJ and Rice JC: Catalytic function of the PR-Set7 histone $\mathrm{H} 4$ lysine 20 monomethyltransferase is essential for mitotic entry and genomic stability. J Biol Chem 283: 19478-19488, 2008.

38. Oda H, Okamoto I, Murphy N, Chu J, Price SM, Shen MM, Torres-Padilla ME, Heard E and Reinberg D: Monomethylation of histone H4-lysine 20 is involved in chromosome structure and stability and is essential for mouse development. Mol Cell Biol 29: 2278-2295, 2009.

39. Jørgensen S, Elvers I, Trelle MB, Menzel T, Eskildsen M, Jensen ON, Helleday T, Helin K and Sørensen CS: The histone methyltransferase SET8 is required for S-phase progression. J Cell Biol 179: 1337-1345, 2007.

40. Centore RC, Havens CG, Manning AL, Li JM, Flynn RL, Tse A, Jin J, Dyson NJ, Walter JC and Zou L: CRL4 $4^{\operatorname{Cdt} 2}$-mediated destruction of the histone methyltransferase Set 8 prevents premature chromatin compaction in S phase. Mol Cell 40: 22-33, 2010.

41. Sanders SL, Portoso M, Mata J, Bähler J, Allshire RC and Kouzarides T: Methylation of histone H4 lysine 20 controls recruitment of Crb2 to sites of DNA damage. Cell 119: 603-614, 2004

42. Oda H, Hübner MR, Beck DB, Vermeulen M, Hurwitz J, Spector DL and Reinberg D: Regulation of the histone H4 monomethylase PR-Set7 by CRL4 ${ }^{\text {Cdt2 }}$-mediated PCNA-dependent degradation during DNA damage. Mol Cell 40: 364-376, 2010.

43. Yu N, Huangyang P, Yang X, Han X, Yan R, Jia H, Shang Y and Sun L: microRNA-7 suppresses the invasive potential of breast cancer cells and sensitizes cells to DNA damages by targeting histone methyltransferase SET8. J Biol Chem 288: 19633-19642, 2013. 